\title{
Modulation of Fatty Acids and Interleukin-6 in Glioma Cells by South American Tea Extracts and their Phenolic Compounds
}

María C. Cittadini, Ignacio García-Estévez, M. Teresa Escribano-Bailón, Julián C. Rivas-Gonzalo, Mirta A. Valentich, Gastón Repossi \& Elio A. Soria

To cite this article: María C. Cittadini, Ignacio García-Estévez, M. Teresa Escribano-Bailón, Julián C. Rivas-Gonzalo, Mirta A. Valentich, Gastón Repossi \& Elio A. Soria (2017): Modulation of Fatty Acids and Interleukin-6 in Glioma Cells by South American Tea Extracts and their Phenolic Compounds, Nutrition and Cancer, DOI: $10.1080 / 01635581.2018 .1412484$

To link to this article: https://doi.org/10.1080/01635581.2018.1412484

View supplementary material $₫$

Published online: 21 Dec 2017.

Submit your article to this journal $₫$

View related articles

View Crossmark data $\nearrow$ 


\title{
Modulation of Fatty Acids and Interleukin- 6 in Glioma Cells by South American Tea Extracts and their Phenolic Compounds
}

\author{
María C. Cittadini ${ }^{\mathrm{a}, \mathrm{b}}$, Ignacio García-Estévez ${ }^{c}$, M. Teresa Escribano-Bailón c, Julián C. Rivas-Gonzaloc, \\ Mirta A. Valentich ${ }^{\mathrm{a}, \mathrm{d}}$, Gastón Repossi ${ }^{\mathrm{a}, \mathrm{d}}$, and Elio A. Soria $\mathbb{1 D}^{\mathrm{a}, \mathrm{d}}$

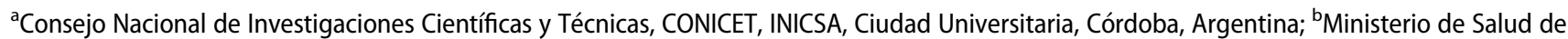 \\ la Nación, Instituto Nacional del Cáncer, CABA, Argentina; 'Universidad de Salamanca, Facultad de Farmacia, Grupo de Investigación en \\ Polifenoles, Campus Miguel de Unamuno, Salamanca, Spain; ${ }^{\mathrm{d} U n i v e r s i d a d ~ N a c i o n a l ~ d e ~ C o ́ r d o b a, ~ F a c u l t a d ~ d e ~ C i e n c i a s ~ M e ́ d i c a s, ~ C a ́ t e d r a ~ d e ~}$ \\ Biología Celular, Histología y Embriología, Instituto de Biología Celular, Córdoba, Argentina
}

\begin{abstract}
Dietary phenolic compounds are plant metabolites with beneficial effects on the central nervous system. Thus, our aim was to identify anti-inflammatory compounds from South American plants on glia, which regulates neuro-immune response. The compounds were extracted from Lantana grisebachii (LG), Aspidosperma quebracho-blanco (AQB), and Ilex paraguariensis (IP) teas and identified by HPLC-DAD-MS. Extracts $(0-200 \mu \mathrm{g} / \mathrm{ml})$ were tested on human T98-G and rat C6 glioma lines. Cellular viability (by the resazurin assay), fatty acid profile (by gas chromatography) and proinflammatory interleukin- 6 release (IL- 6 by ELISA) were determined. Data were analyzed by partial least-square regression to discriminate bioactive compounds. Twenty-one compounds were determined in LG, mainly iridoids, which were linked to $\omega-3$ and $\omega-6$ polyunsaturated fatty acids, but not to IL- 6 release. Thirty-one compounds were found in AQB, mostly hydroxybenzoic derivatives, which were positively related to IL-6 release. Twenty-three compounds were identified in IP, including caffeoylquinic derivatives and mainly chlorogenic acid. They increased the $\omega-7$ palmitoleic fatty acid, which was related to IL-6 decrease. These results enhances phytochemical knowledge of widely available plants, and suggest the lipid-related anti-inflammatory activity of IP phenolic compounds, which give nutritional relevance to the tea.
\end{abstract}

\section{ARTICLE HISTORY}

Received 31 May 2017

Accepted 31 October 2017

\section{Introduction}

Plants are dietary sources of phenolic compounds (or polyphenols), which constitute the largest group of nonenergetic secondary metabolites with redox-modulating activities in mammalian cells $(1,2)$. They are biosynthesized by the shikimic acid and polyacetate pathways, depending on the taxonomic species and on the environmental conditions. The first pathway produces aromatic amino acids, phenolic acids and their derivatives (e.g., hydroxybenzoic and hydroxycinnamic acids, lignans, etc.), while the second one produces quinones and xanthones, among others (1). Aqueous plant extracts contain these compounds, which confer anticancer activity (2).

Three South American plants were selected, Lantana grisebachii Stuck. (Verbenaceae), Aspidosperma quebracho-blanco Schltdl. (Apocynaceae), and Ilex paraguariensis A. St.-Hil. (Aquifoliaceae), because their teas modulate redox homeostasis of the immune and central nervous systems $(3,4)$. They might thus contain molecules with neuro- immune potential to be assayed. Also, these species are accessible for human use given their broad geographical distribution (5), with I. paraguariensis being widely consumed as tea (6). Total phenols and flavonoids have been determined for these plants $(3,4)$, but their detailed phenolic composition was unknown.

The principal cells involved in immune and inflammatory responses in the central nervous system (CNS) are glial cells (7), with gliomas representing the most frequent primary tumors of the CNS (8). They are thus possible targets of polyphenols, which, taken orally, could cross the blood-brain barrier and reach different brain regions (9).

It is also known that phenolic compounds can exert anti-inflammatory activity $(10,11)$, and thus might mitigate the neuroinflammation involved in degenerative, metabolic and neoplastic diseases (11), promoted by proinflammatory mediators, such as interleukin-6 (IL-6) (7). On the other hand, cellular components determine the susceptibility to these responses, with fatty acids playing a recognized role as inflammatory intermediates (11). 
However, precise mechanisms of polyphenol activity are not completely known. Our aim was to identify antiinflammatory phenolic compounds from South American plants on glioma cells.

\section{Materials and Methods}

\section{Plant Extraction}

With the appropriate government consent (Cordoba MINCYT, Argentina), samples of L. grisebachii (LG, $n \geq$ 3) and Aspidosperma quebracho-blanco (AQB, $n \geq 3$ ) were collected during the summer in the South American mountain zone of the phytogeographic Chaco region (GPS coordinates: $-31.28,-64.44)$. Specimens were identified and stored in the RIOC Herbarium (UNRC, Argentina). The $I$. paraguariensis (IP, $n \geq 3$ ) was from organic production in the Parana region. One gram of pulverized air-dried plant (leaves and stems) was extracted by adding $10 \mathrm{ml}$ of $95^{\circ} \mathrm{C}$ water. These infusions (teas) were cooled at room temperature for $1 \mathrm{~h}$, reaching a final temperature of $35-40^{\circ} \mathrm{C}$ (in darkness, with constant agitation). Finally, the extracts were recovered from infusions by filtration (using sterile $0.2-\mu \mathrm{m}$-pore polyethersulfone membranes for 4.5 bars) and then lyophilized, obtaining $0.259 \mathrm{~g}$ of LG extract, $0.194 \mathrm{~g}$ of AQB extract, and $0.497 \mathrm{~g}$ of IP extract, per gram of each plant.

\section{Phytochemistry by HPLC-DAD-MS}

The phenolic composition of the plant extracts was analyzed using a Hewlett-Packard 1100 series liquid chromatograph (Agilent Technologies, Waldbronn, Germany). A Spherisorb ${ }^{\circledR}$ S3 ODS-2 C18 reversedphase, $3 \mu \mathrm{m}, 150 \times 4.6 \mathrm{~mm}$ column (Waters Corporation, Milford, MA, USA) was used with thermostat at $35^{\circ} \mathrm{C}$. The HPLC conditions were optimized for the analysis of these samples. The solvents employed were: (A) an aqueous solution (0.1\%) of formic acid, (B) $100 \%$ HPLC-grade acetonitrile, establishing the following gradient: from $0 \%$ to $10 \%$ B for $3 \mathrm{~min}$, from $10 \%$ to $14.5 \%$ B for $34 \mathrm{~min}$, from $14.5 \%$ to $20 \%$ B for $3 \mathrm{~min}$, from $20 \%$ to $35 \%$ B for $15 \mathrm{~min}$ and from $35 \%$ to $60 \%$ B for $5 \mathrm{~min}$, at a flow rate of $0.5 \mathrm{ml} / \mathrm{min}$. Spectra were recorded from 220 to $600 \mathrm{~nm}$ and detection was carried out at 250,280 , 330 , and $370 \mathrm{~nm}$ as the preferred wavelengths. The mass spectrometer was connected to the HPLC system via the DAD cell outlet. Mass spectrometry was carried out using an API 3200 Qtrap (Applied Biosystems, Darmstadt, Germany) equipped with an ESI source and a triple quadrupole linear ion trap mass analyzer controlled by Analyst 5.1 software. The mass spectrometry was performed in negative mode per the methodology used by
García-Estévez (12) for the analysis of flavanols and phenolic acids: declustering potential $-40 \mathrm{~V}$, entrance potential $-7 \mathrm{~V}$, ion spray voltage $5000 \mathrm{~V}$, GS1 $40 \mathrm{psi}$, GS2 50 psi, curtain gas 20 psi, with collision gas set as "high." Two mass experiments were performed: full mass analysis (collision energy $10 \mathrm{~V}$ ) and MS2 analysis (collision energy 25V). Moreover, to determine the most polar compounds in $\mathrm{AQB}$, its extract was also analyzed using the chromatographic method used by GarcíaEstévez et al. for the analysis of ellagitannins (13) and the corresponding mass spectrometry method for full mass analysis (14).

For the phytochemical characterization of each plant, $1 \mathrm{mg} / \mathrm{ml}$ of dry extract of LG and IP was used and $1.5 \mathrm{mg} / \mathrm{ml}$ of dry extract of $\mathrm{AQB}$, since previous testing indicated the existence of phenolic compounds in low concentrations in the latter. The compounds were identified by their retention times, UV spectra and molecular and fragment ions supplied by the mass spectrometry (MS) analysis. The differences in relative abundance of the fragment ions detected for each compound were used to differentiate between isomers by comparison with the fragmentation patterns reported in literature $(15,16)$. Each identified compound was quantified based on its molecular structure (see section results: Tables 1-3). The standards used to identify and quantify the identified compounds (as bracketed) were gallic acid (compounds 1, 4, 14 of LG; 1-3, 7, 9, 10, 13$18,26,28,30$ of AQB and 2, 3, 12 of IP), protocatechuic acid (compounds 6 and 12 of AQB), chlorogenic acid (compound 3 of LG; 5, 11 of AQB and 1, 4-6, 9, 10, 11, $13,14,16,17,19-23$ of IP), caffeic acid (compounds 9 , $10,12,15-17$ of LG and 7-8 of IP), vanillic acid (compound 4 of $A Q B)$, syringic acid (compound 8 of $A Q B)$, p-coumaric acid (compound 6 of LG), ellagic acid (compound 31 of $\mathrm{AQB}$ ), geniposidic acid (compounds $2,5,7,8,11,13,20,21$ of LG), epicatechin (compound 27 of $\mathrm{AQB}$ ), catechin (compound 24 of $\mathrm{AQB}$ ), quercetin 3-O-glucoside (compound 19 of AQB); quercetin 3-Orutinoside (compound 25 of $\mathrm{AQB}$ and 15 of IP), isorhamnetin 3-O-glucoside (compounds 18-19 of LG and 22-23 of AQB), isorhamnetin 3-O-rutinoside (compound 29 of $\mathrm{AQB}$ and 18 of IP), and kaempferol 3-O-glucoside (compounds 20-21 of AQB).

\section{Cell Culture and Treatment}

Human T98-G cells (ATCC ${ }^{\circledR} \mathrm{n}^{\circ}$ CRL-1690 ${ }^{\mathrm{TM}}$ ) and rat C6 cells $\left(\right.$ ATCC $^{\circledR} \mathrm{n}^{\circ}$ CCL-107 ${ }^{\mathrm{TM}}$ ) were cultured in Dulbecco's modified Eagle medium (DMEM) supplemented with fetal bovine serum (10\%), penicillin G sodium $(100 \mathrm{IU} / \mathrm{ml})$ and gentamycin sulphate $(40 \mathrm{mg} / \mathrm{ml})$, at $37^{\circ} \mathrm{C}$ and $5 \% \mathrm{CO}_{2}$. After these cells were seeded in 
Table 1. Chromatographic characteristics $\left(t_{\mathrm{R}}\right)$, mass spectral data, UV spectrum $\left(\lambda_{\max }\right)$, tentative identification and concentration of phenolic compounds ( $\mathrm{mg} / \mathrm{g}$ of dried extract) in Lantana grisebachii extract.

\begin{tabular}{|c|c|c|c|c|c|c|c|c|}
\hline Compound & $t_{\mathrm{R}}(\min )$ & $\begin{array}{c}{[\mathrm{M}-\mathrm{H}]^{-}} \\
(\mathrm{m} / \mathrm{z})\end{array}$ & Fragment ions $(\mathrm{m} / \mathrm{z})$ & $U V \lambda_{\max }(\mathrm{nm})$ & identification & Group & $\begin{array}{c}\mathrm{mg} / \mathrm{g} \text { of } \\
\text { dried extract }\end{array}$ & $\%$ \\
\hline 1 & 5.56 & 282 & 150,133 & $254,274(\mathrm{sh})$ & unknown & - & 2.94 & 1.75 \\
\hline 2 & 5.86 & 391 & $167,123,149$ & 236 & Dihydrotheveside & Iridoid & 26.75 & 15.91 \\
\hline 3 & 6.24 & 353 & 191 & $326,298(\mathrm{sh})$ & 3-Caffeoylquinic acid & QA derivatives & 1.64 & 0.98 \\
\hline 4 & 8.04 & 375 & $169,151,213$ & 238 & Unknown & - & 3.80 & 2.26 \\
\hline 5 & 9.01 & 373 & $167,211,123,149,193$ & 232,314 & Geniposidic acid & Iridoid & 1.77 & 1.05 \\
\hline 6 & 10.11 & 487 & $179,135,161$ & 332,300 (sh) & Coumaroyl-hesperidoside & HA derivatives & 1.19 & 0.71 \\
\hline 7 & 11.09 & 389 & $345,121,165,209,139$ & 236 & Theveside & Iridoid & 41.69 & 24.79 \\
\hline 8 & 17.36 & 403 & $371,127,191,121,139$ & 234 & Theviridoside & Iridoid & 15.47 & 9.20 \\
\hline 9 & 19.20 & 639 & $621,161,179,459,529$ & 330, 296(sh) & $\beta$ OH-Acteoside ${ }^{*}$ & HA derivatives & 2.93 & 1.74 \\
\hline 10 & 19.49 & 639 & $621,161,179,459,529$ & $330,296(\mathrm{sh})$ & $\beta$ OH-Acteoside ${ }^{*}$ & HA derivatives & 3.04 & 1.81 \\
\hline 11 & 23.18 & 537 & $163,373,313,331,119,149$ & 232, 296(sh), 312 & Coumaroyl-geniposidic acid & Iridoid & 3.71 & 2.20 \\
\hline 12 & 23.51 & 623 & $461,161,315,179$ & 246, 294(sh), 332 & Verbascoside isomer & HA derivatives & 20.56 & 12.22 \\
\hline 13 & 24.58 & 567 & $193,373,343,149,361$ & $326,296(\mathrm{sh})$ & Feruloyl-geniposidic acid & Iridoid & 4.59 & 2.73 \\
\hline 14 & 25.08 & 637 & 315,301 & 364,300 (sh) & $\begin{array}{l}\text { Isorhamnetin O-rhamnosyl- } \\
\text { glucuronide }\end{array}$ & Flavonol & 4.84 & 2.88 \\
\hline 15 & 25.83 & 623 & $461,161,315$ & 328, 292(sh) & Verbascoside isomer & HA derivatives & 2.15 & 1.28 \\
\hline 16 & 26.19 & 623 & $461,161,315,179$ & 328, 292(sh) & Verbascoside isomer & HA derivatives & 1.96 & 1.17 \\
\hline 17 & 26.52 & 623 & 461,161 & 328,292 (sh) & Verbascoside isomer & HA derivatives & 2.89 & 1.72 \\
\hline 18 & 26.89 & 491 & 315 & 360,299 (sh) & $\begin{array}{l}\text { Isorhamnetin 3-O- } \\
\text { glucuronide }\end{array}$ & Flavonol & 6.67 & 3.96 \\
\hline 19 & 27.46 & 477 & 315 & 362, 299 (sh) & Isorhamnetin 3-O-glucoside & Flavonol & 8.51 & 5.06 \\
\hline 20 & 28.42 & 557 & $373,149,133$ & 230,326 & Geniposidic acid derivative & Iridoid & 5.55 & 3.30 \\
\hline 21 & 30.12 & 559 & $373,149,185$ & 230, 296(sh), 330 & Geniposidic acid derivative & Iridoid & 5.53 & 3.29 \\
\hline
\end{tabular}

${ }^{*}$ Hydroxylated verbascoside.

QA: Quinic acid derivatives.

HA. Hydroxycinnamic acid derivatives.

sterile clear flat bottom polystyrene $96-$ well plates $(50,000$ cells $/ \mathrm{cm}^{2}$ ) and incubated for $24 \mathrm{~h}$ to achieve maximum attachment, they were treated for $72 \mathrm{~h}$ with LG, AQB or IP (dose range: 0 -control-, 50, 100, $200 \mu \mathrm{g} / \mathrm{ml}$ ), with each phenolic compound being quantified and used as statistical predictor. Eight separate experiments were performed in triplicate assaying each one of the three plant extracts.

\section{Cellular Viability}

Cellular viability was measured and confirmed by the resazurin-based assay. Briefly, viable cells were stained with resazurin $(0.05 \mathrm{mg} / \mathrm{ml}$ in culture medium for $6 \mathrm{~h})$ to record absorbance at $600 \mathrm{~nm}$ (GloMax-Multi microplate reader, Promega Corp., USA) (17). Cellular viability was a necessary condition to release IL- 6 and consequently included in statistical models.

\section{Gas Chromatography of Fatty Acids}

Total lipids were extracted and partitioned from cells placed in PBS by the Folch method (18). The phase containing phospholipids was treated with sodium methoxide at $4^{\circ} \mathrm{C}$ overnight to obtain fatty acid methyl esters, which were extracted and dried with nitrogen (19). The separation, quantification and identification of esters were performed using a capillary column (BPX $20 \mathrm{~m}$ longitude, $0.25 \mathrm{~mm}$ ID, $0.25 \mu \mathrm{m}$ film, SUPELCO ${ }^{\odot}$, USA) in a Clarus $500^{\odot}$ (Perkin-Elmer ${ }^{\circledR}$ ) gas-liquid chromatograph with a flame ionization detector (19). Fatty acids (FA) were grouped into their $\omega$ families: saturated FA: myristic (14:0), palmitic (16:0), stearic (18:0), lignoceric (24:0); $\omega 3$ FA: $\alpha$-linoleic (18:3), eicosatrienoic (20:3), eicosapentaenoic (20:5), docosapentaenoic (22:5), docosahexaenoic (22:6). $\omega 6$ FA: linoleic (18:2), $\gamma$-linoleic (18:3), eicosadienoic (20:2), arachidonic (20:4). $\omega 7$ FA: palmitoleic (16:1). $\omega 9$ FA: oleic (18:1), eicosanoic (20:1), erucic (22:1), nervonic (24:1). Their percentage amounts were used as continuous variables in statistical models.

\section{IL-6 Determination}

IL-6 was measured in media by immuno-ELISA (450 and $570 \mathrm{~nm}$ ) by a commercial kit (BD Biosciences) (7). This interleukin was a response variable in statistical models.

\section{Statistical Analysis}

Extract doses and cellular responses were analyzed by linear regression in both cell lines. Partial least squares regression (PLS) was used to discriminate compound effects and response relations, by modelling compound doses as predictors, cellular viability, fatty acids and IL-6 release as responses, and cell lines C6 and T98 as categorical data (Infostat v.2012 software). This method reports standardized correlations to be graphed by linear charts $(20,21)$. 
Table 2. Chromatographic characteristics $\left(t_{\mathrm{R}}\right)$, mass spectral data, UV spectrum $\left(\lambda_{\text {max }}\right)$, tentative identification and concentration of phenolic compounds ( $\mathrm{mg} / \mathrm{g}$ of dried extract) in Aspidosperma quebracho blanco extract.

\begin{tabular}{|c|c|c|c|c|c|c|c|c|}
\hline Compound & $t_{\mathrm{R}}(\min )$ & $\begin{array}{c}{[\mathrm{M}-\mathrm{H}]^{-}} \\
(\mathrm{m} / \mathrm{z})\end{array}$ & Fragment ions $(\mathrm{m} / \mathrm{z})$ & UV $\lambda_{\max }(\mathrm{nm})$ & Identification & Group & $\begin{array}{c}\mathrm{mg} / \mathrm{g} \text { of } \\
\text { dried extract }\end{array}$ & $\%$ \\
\hline $1^{*}$ & 7.31 & 295 & 161 & 260 & unknown & - & 0.71 & 0.80 \\
\hline $2^{*}$ & 7.43 & 237 & $191,135,121$ & 250 & unknown & - & 4.80 & 5.40 \\
\hline 3 & 7.54 & 315 & 153,109 & 240,306 & Gentisoyl-hexoside & HBA derivatives & 2.52 & 2.83 \\
\hline $4^{*}$ & 8.22 & 151 & 109,133 & 268 & Vanillin & HBA derivatives & 1.21 & 1.35 \\
\hline 5 & 8.62 & 353 & & & Chlorogenic acid & QA derivatives & 0.37 & 0.42 \\
\hline 6 & 8.91 & 315 & 153,109 & 260,294 & Protocatechuic acid-hexoside & HBA derivatives & 0.66 & 0.74 \\
\hline $7^{*}$ & 9.08 & 169 & 125 & 270 & Gallic acid & HBA derivatives & 6.70 & 7.52 \\
\hline $8^{*}$ & 9.79 & 197 & 153,135 & 274 & Syringic acid & HBA derivatives & 0.36 & 0.40 \\
\hline $9^{*}$ & 11.05 & 389 & $345,121,165,209$ & 234,326 & Phthalic acid derivative & HBA derivatives & 0.50 & 0.56 \\
\hline $10^{*}$ & 12.99 & 331 & 169,151 & 271 & Galloyl-glucose & HBA derivatives & 0.14 & 0.16 \\
\hline 11 & 13.72 & 353 & 191 & $326,298(\mathrm{sh})$ & 1-Caffeoylquinic acid & QA derivatives & 10.89 & 12.24 \\
\hline $12^{*}$ & 14.62 & 153 & 109 & 259,294 & Protocatechuic acid & HBA derivatives & 2.12 & 2.38 \\
\hline $13^{*}$ & 15.4 & 315 & 153,109 & 263,317 & Gentisoyl-hexoside & HBA derivatives & 6.66 & 7.49 \\
\hline 14 & 15.56 & 403 & $357,125,151,191$ & 246 & unknown & - & 4.34 & 4.88 \\
\hline $15^{*}$ & 16.74 & 375 & $213,169,107$ & & Trihydroxy-phthalic acid hexoside & HBA derivatives & 7.34 & 8.24 \\
\hline 16 & 17.36 & 371 & $249,121,353,231$ & 232,274 & unknown & - & 3.28 & 3.69 \\
\hline 17 & 17.80 & 403 & $371,121,191,223$ & 236 & unknown & - & 5.24 & 5.89 \\
\hline $18^{*}$ & 18.54 & 477 & 153,109 & & Gentisoyl-dihexoside & HBA derivatives & 6.69 & 7.51 \\
\hline 19 & 19.61 & 771 & 301 & 364,300 (sh) & Quercetin glucosyl-rhamnosyl-glucoside & Flavonol & 3.73 & 4.20 \\
\hline 20 & 21.38 & 775 & 285 & 365,300 (sh) & Kaempferol glucosyl-rhamnosyl-galactoside & Flavonol & 1.05 & 1.18 \\
\hline 21 & 21.53 & 775 & 285 & $364,298(\mathrm{sh})$ & Kaempferol glucosyl-rhamnosyl-glucoside & Flavonol & 1.85 & 2.08 \\
\hline 22 & 21.70 & 785 & 315 & 361,302 (sh) & Isorhamnetin glucosyl-rhamnosyl-galactoside & Flavonol & 2.16 & 2.43 \\
\hline 23 & 21.87 & 785 & 315 & 361,302 (sh) & Isorhamnetin glucosyl-rhamnosyl-glucoside & Flavonol & 1.63 & 1.83 \\
\hline 24 & 23.63 & 289 & 245,137 & 278 & Catechin & Flavanol & 0.76 & 0.85 \\
\hline 25 & 24.42 & 609 & 301 & 368 & Quercetin rutinoside & Flavonol & 2.20 & 2.47 \\
\hline 26 & 25.80 & 507 & $463,153,353$ & 306 & unknown & - & 1.86 & 2.09 \\
\hline 27 & 27.40 & 289 & $245,137,151$ & 270 & Epicatechin & Flavanols & 1.34 & 1.51 \\
\hline 28 & 27.50 & 417 & $181,387,167,151,403$ & 262,306 & unknown & - & 4.68 & 5.26 \\
\hline 29 & 29 & 623 & 315 & $362,298(\mathrm{sh})$ & Isorhamnetin 3-O-rutinoside & Flavonol & 0.93 & 1.05 \\
\hline 30 & 35.12 & 389 & $181,275,345,285$ & $274,308,376$ & unknown & - & 1.37 & 1.53 \\
\hline 31 & 38.73 & 301 & $257,153,109$ & 268,344 & Ellagic acid & HBA derivatives & 0.93 & 1.05 \\
\hline
\end{tabular}

*These compounds were determined from the chromatogram obtained in the analysis of the sample using the chromatographic method described in GarcíaEstévez et al. (REF) (see section HPLC).

HBA: Hydroxybenzoic acid derivatives.

QA: Quinic acid derivatives.

\section{Results}

\section{Phytochemistry}

The phenolic composition results were quantified in $\mathrm{mg} /$ $\mathrm{g}$ of plant and then converted into $\mathrm{mg} / \mathrm{g}$ of dry extract (Tables 1-3).

LG: The phenolic profile of LG (Table 1) showed high concentrations of iridoid glycosides, which reached 50\% of the total phenolic compounds identified (compounds 2, 5, 7-8, 11, 13, 20-21). Compound 7 was the most abundant in this sample, reaching $10.4 \mathrm{mg} / \mathrm{g}$. It was identified as the iridoid theveside, based on its UV spectra and on the results of the mass analysis, which showed a pseudomolecular ion at $\mathrm{m} / z 389$ with a fragmentation pattern with a significant peak at $m / z 345$. Compound 2 was detected at a level of $6.93 \mathrm{mg} / \mathrm{g}$ of plant and was identified as dihydrotheveside (pseudomolecular ion at $\mathrm{m} / \mathrm{z} 391)$. The third most abundant compound $(4.01 \mathrm{mg} /$ $\mathrm{g}$ of plant) was theviridoside (compound 8), which showed a $[\mathrm{M}-\mathrm{H}]^{-}$ion at $\mathrm{m} / z$ 403. These iridoid glycosides exhibited UV spectra with a maximum $\left(\lambda_{\max }\right)$ at $\sim 234$ to $236 \mathrm{~nm}$.
Compound 5 was identified as geniposidic acid, which was found at a lower amount of $0.45 \mathrm{mg} / \mathrm{g}$ of plant. Based on the mass spectrometric features of this compound (Table 1), compounds 11 and 13, which showed deprotonated molecular ions at $\mathrm{m} / z 537$ and $\mathrm{m} / \mathrm{z}$ 567, respectively, were identified. Compound 11 showed a fragmentation pattern with major peaks at $\mathrm{m} / \mathrm{z} 163$ ([coumaric acid-H $]^{-}$) and 373 ([geniposidic acid-H $]^{-}$), identifying it as coumaroyl-geniposidic acid $(0.96 \mathrm{mg} / \mathrm{g}$ of plant). Similarly, compound 13 showed major fragment ions at $m / z 193$ ([ferulic acid-H] $]^{-}$) and $m / z 373$

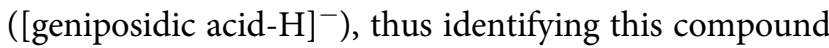
as feruloyl-geniposidic acid (1.19 $\mathrm{mg} / \mathrm{g}$ of plant). Compounds $20\left(t_{\mathrm{R}} 28.42 \mathrm{~min}\right.$, pseudomolecular ion at $\mathrm{m} / z$ 557) and compound $21\left(t_{\mathrm{R}} 30.12 \mathrm{~min}\right.$, pseudomolecular ion at $\mathrm{m} / z$ 559), with fragment ions at $\mathrm{m} / z 373$ and $m / z 149$, were also identified as geniposidic derivatives, although their identity was not fully determined. These compounds were detected at levels of $1.44 \mathrm{mg} / \mathrm{g}$ and $1.43 \mathrm{mg} / \mathrm{g}$ of plant, respectively.

Compounds 6, 9, 10, 12, 15-17, showed UV spectra characteristics of hydroxycinnamic acids. Among these, 
Table 3. Chromatographic characteristics $\left(t_{\mathrm{R}}\right)$, mass spectral data, UV spectrum $\left(\lambda_{\max }\right)$, tentative identification and concentration of phenolic compounds (mg/g of dried extract) in llex paraguariensis extract.

\begin{tabular}{|c|c|c|c|c|c|c|c|c|}
\hline Compound & $\begin{array}{c}t_{R} \\
(\min )\end{array}$ & $\begin{array}{c}{[\mathrm{M}-\mathrm{H}]^{-}} \\
(\mathrm{m} / \mathrm{z})\end{array}$ & Fragment ions $(m / z)$ & $\begin{array}{l}\text { UV } \lambda_{\max } \\
(\mathrm{nm})\end{array}$ & Identification & Group & $\begin{array}{c}\mathrm{mg} / \mathrm{g} \text { of } \\
\text { dried extract }\end{array}$ & $\%$ \\
\hline 1 & 4.47 & 191 & 111,129 & 266 & Quinic acid & QA derivatives & 3.28 & 1.38 \\
\hline 2 & 4.88 & 371 & $191,173,135$ & 252,278 & Unknown & - & 0.59 & 0.25 \\
\hline 3 & 5.018 & 473 & $189,351,221,311$ & 294 & Unknown & - & 0.61 & 0.26 \\
\hline 4 & 7.41 & 353 & 191(100), 179(49), 135(27) & 326,298 (sh) & 3-Caffeoylquinic acid & QA derivatives & 2.01 & 0.85 \\
\hline 5 & 8.04 & 353 & $191(100), 179(50), 135(30)$ & $326,296(\mathrm{sh})$ & 5-Caffeoylquinic ${ }^{1}$ & QA derivatives & 57.80 & 24.37 \\
\hline 6 & 8.50 & 391 & $217,179,135$ & 272 & 3-Disuccinoyl quinic acid & QA derivatives & 7.44 & 3.13 \\
\hline 7 & 8.83 & 341 & $161,179,203,135$ & 328,300 (sh) & Caffeoyl acid glucose & HA derivatives & 2.39 & 1.01 \\
\hline 8 & 10.67 & 341 & $179,161,221,135$ & 328,300 (sh) & Caffeoyl acid glucose & HA derivatives & 2.06 & 0.87 \\
\hline 9 & 12.33 & 353 & 173(100), 179(90), 191(75), 135(70) & $326,298(\mathrm{sh})$ & 4-Caffeoyl quinic acid & QA derivatives & 23.49 & 9.91 \\
\hline 10 & 12.89 & 367 & 193(100), 134(15) & $324,294(\mathrm{sh})$ & 3-Feruloylquinic acid & QA derivatives & 4.40 & 1.85 \\
\hline 11 & 13.31 & 353 & 191(100), 179(4) & 326,300 (sh) & 1-Caffeoylquinic acid & QA derivatives & 35.52 & 14.98 \\
\hline 12 & 16.96 & 427 & 161,179 & 274 & Unknown & - & 12.99 & 5.48 \\
\hline 13 & 18.43 & 367 & 173(100), 191(29) & 322,292 (sh) & 4-Feruloylquinic acid & QA derivatives & 2.37 & 0.99 \\
\hline 14 & 20.12 & 367 & 191(100), 173(12) & $324,296(\mathrm{sh})$ & 5-Feruloylquinic acid & QA derivatives & 2.99 & 1.26 \\
\hline 15 & 24.49 & 609 & 301 & 364,300 (sh) & Quercetin rutinoside & Flavonol & 12.36 & 5.21 \\
\hline 16 & 26.12 & 515 & $353(100), 173(60), 335(20)$ & 326,298 (sh) & 3,4-Dicaffeoylquinic acid & QA derivatives & 8.62 & 3.63 \\
\hline 17 & 27.80 & 515 & 353(100), 191(80), 179(60) & 328,298 (sh) & 1,3-Dicaffeoylquinic acid & QA derivatives & 37.43 & 15.78 \\
\hline 18 & 29.18 & 623 & 315 & $362,300(\mathrm{sh})$ & Isorhamnetin rutinoside & Flavonol & 0.69 & 0.29 \\
\hline 19 & 30.66 & 515 & $\begin{array}{c}353(100), 173(90), 179(70) \\
191(50), 203(20)\end{array}$ & $328,300(\mathrm{sh})$ & 1,4-Dicaffeoylquinic acid & QA derivatives & 14.03 & 5.92 \\
\hline 20 & 34.53 & 529 & $367(100), 193(80), 134(20)$ & $324,294(\mathrm{sh})$ & 3,5-Caffeoyl-feruloylquinic ${ }^{2}$ & QA derivatives & 1.86 & 0.79 \\
\hline 21 & 35.15 & 529 & $353(100), 191(80), 179(60)$ & $324,294(\mathrm{sh})$ & 3,5-Caffeoyl-feruloylquinic ${ }^{3}$ & QA derivatives & 1.32 & 0.56 \\
\hline 22 & 35.55 & 515 & $353(100), 173(70), 179(50)$ & $326,298(\mathrm{sh})$ & 4-5 Dicaffeoylquinic acid & QA derivatives & 1.25 & 0.53 \\
\hline 23 & 37.19 & 529 & $367(100), 173(80)$ & 328,300 (sh) & 3,4-Caffeoyl-feruloylquinic ${ }^{4}$ & QA derivatives & 1.67 & 0.70 \\
\hline
\end{tabular}

QA: Quinic acid derivatives; HA: hydroxycinnamic acid derivatives.

${ }^{1}$ Chlorogenic acid; 2 3,5-Caffeoyl-feruloylquinic acid(3F,5C).

3 3,5-Caffeoyl-feruloylquinic acid(3C,5F).

4 3,4-Caffeoyl-feruloylquinic acid(3C,4F).

compounds 12 and 15-17 were identified as verbascoside isomers. Compound $\mathbf{1 2}$ was the most abundant, reaching $5.33 \mathrm{mg} / \mathrm{g}$ of plant. These four compounds presented the same UV spectra and the same pseudomolecular ion $\left([\mathrm{M}-\mathrm{H}]^{-}\right)$at $m / z 623$, with similar fragmentation patterns (fragments ions at $\mathrm{m} / z$ 461, 161, 315, and/or 179). Compounds 9 and 10 were identified as the hydroxylated verbascoside called $\beta \mathrm{OH}$-acteoside $(0.76 \mathrm{mg} / \mathrm{g}$ of plant and $0.79 \mathrm{mg} / \mathrm{g}$ of plant, respectively). Its molecular ion was found at $m / z 639$ with a major fragment ion at $\mathrm{m} / \mathrm{z} 621$, followed by another at $m / z 161$.

Flavonols were also identified in LG. Compounds 18 and 19 showed pseudomolecular ions at $\mathrm{m} / \mathrm{z} 491$ and 477 , respectively, with a major fragment ion at $\mathrm{m} / z 315$ $\left([\text { isorhamnetin-H }]^{-}\right)$, i.e., losses of 176 and 162, respectively. Therefore, taking into account that the normal position of glycosylation of flavonols is the hydroxyl group in the C-3, they were identified as isorhamnetin 3$O$-glucuronide $(1.73 \mathrm{mg} / \mathrm{g}$ of plant) and isorhamnetin 3-O-glucoside $(2.20 \mathrm{mg} / \mathrm{g}$ of plant), respectively. Compound 14, which showed a pseudomolecular ion at $m / z 637$ and a major fragment ion at $m / z 315$ ([isorhamnetin- $\mathrm{H}]^{-}$), was identified as isorhamnetin rhamnosylglucuronide (1.25 mg/g of plant). All these compounds showed the characteristic UV spectrum of flavonols (Table 1). Only compound 3 was identified as a quinic derivative, since its molecular ion was detected at $\mathrm{m} / \mathrm{z}$
353 with fragment ion at $m / z 191$. It was denominated 3caffeoylquinic acid $(0.42 \mathrm{mg} / \mathrm{g}$ of plant).

AQB: In AQB, 31 phenolic compounds were identified (Table 2), with hydroxybenzoic derivatives predominating (compounds 3, 4, 6-10, 12, 13, 15, 18, 31).

Compounds 3 and 13, defined as gentisoyl hexoside (0.49 $1.29 \mathrm{mg} / \mathrm{g}$ of plant, respectively), showed a pseudomolecular ion at $m / z 315$, with fragment ions at $m / z 153$ ([gentisic acid-H] $]^{-}$) and $m / z$ 109. Compound 18, showing a pseudomolecular ion at $m / z 477$ and the same fragmentation pattern $\left.(\mathrm{m} / z 153 \text { ([gentisic acid-H] }]^{-}\right)$and $\mathrm{m} / z$ 109) was identified as gentisoyl dihexoside $(1.30 \mathrm{mg} / \mathrm{g}$ of plant). Compound 9 (molecular ion at $m / z$ 389) and compound 15 (molecular ion at $\mathrm{m} / z$ 375) presented characteristics of phthalic derivatives, the latter being identified as trihydroxy-phthalic acid hexoside (respective concentrations: 0.10 and $1.43 \mathrm{mg} / \mathrm{g}$ of plant).

Within hydroxybenzoic acid derivatives, protocatechuic acid was identified (compound 12) showing a pseudomolecular ion at $m / z 153\left([\mathrm{M}-\mathrm{H}]^{-}\right)$, and compound 6 was identified as protocatechuic acid hexoside $\left([\mathrm{M}-\mathrm{H}]^{-}\right)$at $m / z 315$ with a main fragment ion at $m / z$

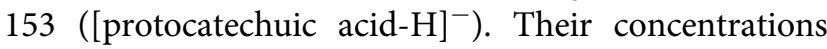
were 0.41 and $0.13 \mathrm{mg} / \mathrm{g}$ of plant, respectively.

Compound 7 was identified as gallic acid $\left([\mathrm{M}-\mathrm{H}]^{-}\right.$at $\mathrm{m} / z$ 169). Its concentration was $1.30 \mathrm{mg} / \mathrm{g}$ of plant. Similarly, compound 10, identified as galloyl glucose $(\mathrm{M}-\mathrm{H}]^{-}$at $m / z 331$ with main fragment ions at $m / z 169$ 
([gallic acid- $\mathrm{H}]^{-}$) and at $\mathrm{m} / z 151$ ([gallic acid- $\left.\mathrm{H}_{2} \mathrm{O}\right]^{-}$), was also found but in lower amounts $(0.03 \mathrm{mg} / \mathrm{g}$ of plant).

Other compounds identified as among hydroxybenzoic acids were compounds 4,8 , and 31 . At $t_{\mathrm{R}} 8.21 \mathrm{~min}$, compound 4 showed a $[\mathrm{M}-\mathrm{H}]^{-}$at $m / z 151$ and was identified as vanillin $(0.23 \mathrm{mg} / \mathrm{g}$ of plant). Syringic acid (compound 8, $0.07 \mathrm{mg} / \mathrm{g}$ of plant) was identified by its pseudomolecular ion at $m / z 197\left([\mathrm{M}-\mathrm{H}]^{-}\right)$and its fragmentation pattern (fragment ions at $\mathrm{m} / z 153$ and 135). Finally, ellagic acid (compound 31, ([M-H] $]^{-}$at $m / z 301$, with fragment ions at $m / z 257,153$, and 109) reached $0.18 \mathrm{mg} / \mathrm{g}$ of plant.

Two compounds of AQB extract were identified as quinic acid derivatives. Compound 5, at a concentration of $0.07 \mathrm{mg} / \mathrm{g}$ of plant, was defined as chlorogenic acid (5caffeoylquinic acid) based on its retention time and on its pseudomolecular ion at $\mathrm{m} / z 353\left([\mathrm{M}-\mathrm{H}]^{-}\right)$. Compound 11 was identified as 1-caffeoyl-quinic acid because its molecular ion was $m / z 353$ with fragment ion at $m / z 191$.

Several compounds identified in AQB (compounds 19-23, 25, and 29) exhibited characteristics of flavonols (spectra UV $\lambda_{\max } \sim 360 \mathrm{~nm}$ ). Compounds 19 and 25 showed a pseudomolecular ion at $m / z 771$ and 609, respectively, and in both cases, the main fragment ion

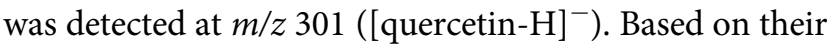
fragmentation patterns, they were defined as quercetin 3 -O-glucosyl-rhamnosyl-glucoside $(0.73 \mathrm{mg} / \mathrm{g}$ of plant $)$ and quercetin 3 -O-rutinoside $(0.43 \mathrm{mg} / \mathrm{g}$ of plant), respectively. In turn, considering their relative chromatographic retention times, UV-vis spectra and mass spectrometry behavior, compounds 20 and 21 were identified as kaempferol 3-O-glucosyl-rhamnosyl-galactoside (from the first chromatographic elution) and kaempferol 3-O-glucosyl-rhamnosyl-glucoside, which showed a pseudomolecular ion at $m / z 775$ with a main $\mathrm{MS}^{2}$ fragment ion at $m / z 285$. Their concentrations were 0.20 and $0.36 \mathrm{mg} / \mathrm{g}$ of plant. Compounds $22(0.42 \mathrm{mg} / \mathrm{g}$ of plant) and $23(0.32 \mathrm{mg} / \mathrm{g}$ of plant $)$ showed a molecular ion at $\mathrm{m} / z 785$ with a main fragment ion at $\mathrm{m} / z 315$ ([isorhamnetin- $\left.\mathrm{H}]^{-}\right)$. They were isorhamnetin 3-O-glucosyl-rhamnosyl-galactoside and isorhamnetin 3-O-glucosyl-rhamnosyl-glucoside (flavonols), respectively, according to their fragmentation pattern and chromatographic retention time. Compound 29, which presented a pseudomolecular ion at $m / z 623$ with $\mathrm{MS}^{2}$ fragments at $m / z 315$ ([isorhamnetin- $\mathrm{H}]^{-}$), was identified as isorhamnetin 3-O-rutinoside $(0.18 \mathrm{mg} / \mathrm{g}$ of plant).

Monomeric flavanols were also found in the AQB. Compound 24, showing a pseudomolecular ion at $\mathrm{m} / \mathrm{z}$ 289 and fragment ions at $\mathrm{m} / z 245$ and 137, was identified as catechin $(0.15 \mathrm{mg} / \mathrm{g}$ plant), and compound 27 as epicatechin $(0.26 \mathrm{mg} / \mathrm{g}$ of plant), which showed the same molecular ion and fragment ions at $m / z 245,137$, and 151.

IP: Most of the phenolic compounds (compounds 1, 4-6, 9-11, 13, 14, 16-23) in this plant showed the UV spectrum characteristic of quinic acid derivatives $\left(\lambda_{\text {máx }}\right.$ $\sim 326 \mathrm{~nm}$ ) (Table 3). Compounds 4, 5, 9, and 11 showed a pseudomolecular ion at $\mathrm{m} / z 353$ and were identified as caffeoylquinic acid isomers. Their identities were assigned depending on the different relative intensities of fragmentation (22). Compound 4 showed fragment ions at $m / z$ 191(100), 79(49), 135(27) and was seen to be 3-caffeoylquinic acid (1 mg/g of plant), and compound 5, which showed fragment ions at $\mathrm{m} / \mathrm{z} 191(100)$, 179(5), and 135(3), was 5-caffeoylquinic acid (chlorogenic acid). This phenolic compound was the most abundant $(28.82 \mathrm{mg} / \mathrm{g}$ of plant). Compound 11 also showed fragments of 191(100) and 179(4) and, given the absence of other fragments, was identified as 1-caffeoylquinic acid ( $17.71 \mathrm{mg} / \mathrm{g}$ of plant). Finally compound 9 showed greater intensity in fragment 173(100), 179(90), 135(70), and lowest in 191(75), and therefore was identified as 4caffeoylquinic acid ( $11.71 \mathrm{mg} / \mathrm{g}$ of plant).

Compounds 16, 17, 19, and 22 presented a pseudomolecular ion at $\mathrm{m} / z 515$ and a main fragment ion at $\mathrm{m} /$ $z$ 353(100), which can correspond to the characteristics of dicaffeoylquinic acids. Given their different intensities of fragmentation, they were identified by comparing them with those reported in literature $(15,16)$. Compound 16, which showed fragment ions at $\mathrm{m} / z$ 353(100), 173(60), 335(20), was defined as 4,3-dicaffeoylquinic acid $(4.3 \mathrm{mg} / \mathrm{g}$ of plant), and compound 17 , with fragment ions at $m / z 353(100), 191(80), 179(60)$, was 1,3 dicaffeoylquinic acid (18.66 mg/g of plant). Compound 19 also showed fragmentation of $m / z$ 353(100), 173(90), 179(70), 191(50), 203(20), and it was identified as 1,4dicaffeoylquinic acid $(6.99 \mathrm{mg} / \mathrm{g}$ of plant). Finally, compound 22 was defined as 4,5-dicaffeoylquinic acid $(0.62 \mathrm{mg} / \mathrm{g}$ of plant) based on its fragmentation pattern (fragment ions at $m / z 353(100), 173(70)$, and 179(50)).

Compounds 10, 13 and 14 showed a pseudomolecular ion at $m / z 367$, which is characteristic of feruloylquinic acids. Based on their fragmentation patterns $(15,16)$, they were identified, respectively, as 3 -feruloylquinic acid (fragments ions at $\mathrm{m} / z$ 193(100) and 134 (15)), 4-feruloylquinic acid (fragment ions at $\mathrm{m} / z 173$ (100) and 191(29)) and 5-feruloylquinic acid $\left(\mathrm{MS}^{2}\right.$ ions at $m / z 191(100)$ and 173(12)). The observed amounts of each isomer were $2.19,1.18$, and $1.49 \mathrm{mg} / \mathrm{g}$ of plant, respectively.

Compounds 20, 21, and 23 showed a molecular ion at $m / z 529$, which can be related to caffeoyl-feruloylquinic acid structures. The different isomers were 
identified by comparing their $\mathrm{MS}^{2}$ fragmentation pattern with those previously reported $(15,16)$. Compound 20, identified as 3,5-caffeoyl-feruloylquinic acid (3F, 5C), showed fragment ions at $m / z 367(100), 193(80)$ ([ferulic acid- $\mathrm{H}]^{-}$) and $134(20)$ and its concentration was $0.93 \mathrm{mg} / \mathrm{g}$ of plant. Compound 21 was identified as 3,5caffeoyl-feruloylquinic acid (3C,5F), since it showed fragment ions at $\mathrm{m} / z$ 353(100) ([(caffeoylquinic acid$\mathrm{H}]^{-}$), 191(80) ([quinic acid-H $]^{-}$) and 179(60) ([caffeic acid- $\mathrm{H}]^{-}$). Its concentration was $0.66 \mathrm{mg} / \mathrm{g}$ of plant. Finally, compound 23, with values of $0.83 \mathrm{mg} / \mathrm{g}$ of plant, was defined as 3,4-caffeoyl-feruloylquinic acid (3C, 4F) because of its fragmentation pattern $(\mathrm{m} / z$ 367(100) and 173(80)).

Compounds 7 and 8, identified as hydroxycinnamic derivatives, were defined as caffeoyl acid glucose (1.19 and $1.03 \mathrm{mg} / \mathrm{g}$ of plant, respectively). These presented $[\mathrm{M}-\mathrm{H}]^{-}$at $m / z 341$ and $\mathrm{MS}^{2}$ fragmentation at $m / z 179$, 161 , and 135.

Flavonols were also found in the extract of $I P$. Compound 15 showed a molecular ion at $m / z 609$ with a

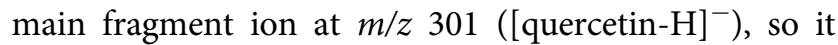
was defined as quercetin rutinoside $(6.16 \mathrm{mg} / \mathrm{g}$ of plant). Similarly, compound $\mathbf{1 8}$ was defined as isorhamnetin rutinoside $(0.34 \mathrm{mg} / \mathrm{g}$ of plant) based on its molecular ion at $m / z 623$ and fragment ion at $m / z 315$ ([isorhamnetin- $\mathrm{H}]^{-}$).

\section{Bioactivity}

Cellular viability of C6 and T98-G lines did not show a significant response to the extracts (AQB, IP, and LG) in the linear regression analysis; thus, they did not show toxicity (Figure 1).

The AQB extract induced IL-6 release by both cell lines in a dose-dependent manner, whereas the LG extract reduced IL-6 release by C6 cells in a non-significant way, without activity on T98-G cells. The IP extract significantly reduced IL-6 release by C6 cells, without activity on T98-G cells. Thus, AQB was pro-inflammatory, LG was not active, and IP was anti-inflammatory (Figure 1).

The lipid profile of C6 cells treated with LG included $\omega-9$, saturated, $\omega-6$, and $\omega-3$ fatty acids in decreasing amounts. Similar results were found in AQB-treated cells, although they showed higher amounts of the first ones ( $\omega-9$, and saturated fatty acids). The $\omega-7$ fatty acids were the major lipids in IP-treated cells, followed by $\omega-9$, saturated, $\omega-6$, and $\omega-3$ acids (Figure 2).

On the other hand, T98-G cells treated with LG mainly showed $\omega-9$ acids followed by $\omega-6, \omega-7$, saturated, and $\omega-3$ ones. IP and AQB enhanced the $\omega-9$ content, followed by $\omega-7$, saturated, $\omega-6$, and $\omega-3$ ones (Figure 2).

PLS regression was used to correlate responses of both cell lines exposed to extract compounds, and to

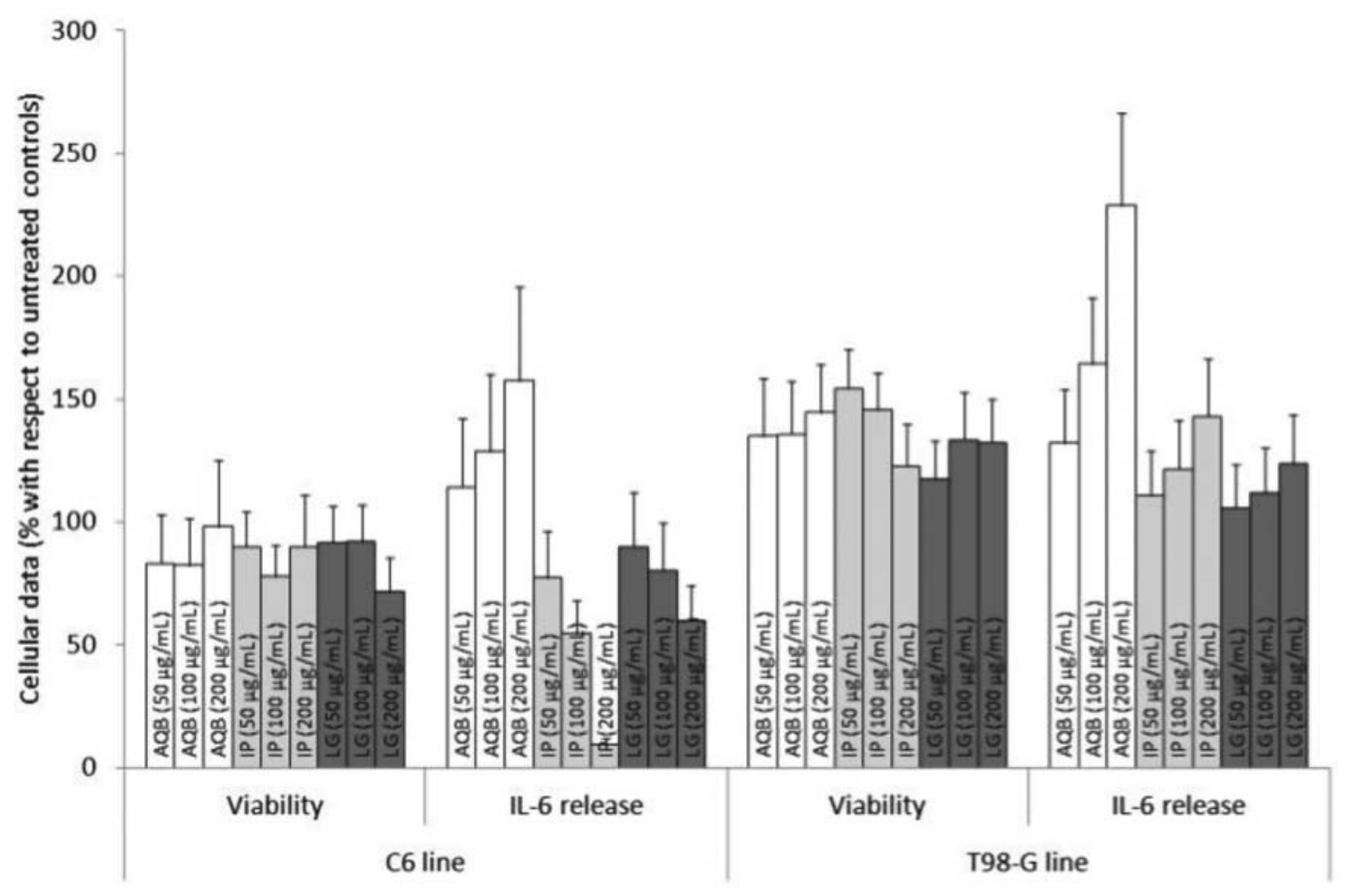

Figure 1. Cellular viability and IL-6 release of the C6 and T98-G cell lines treated with LG, AQB, and IP (expressed as mean percentage and standard error with respect to untreated controls: $C=100 \%)$. 


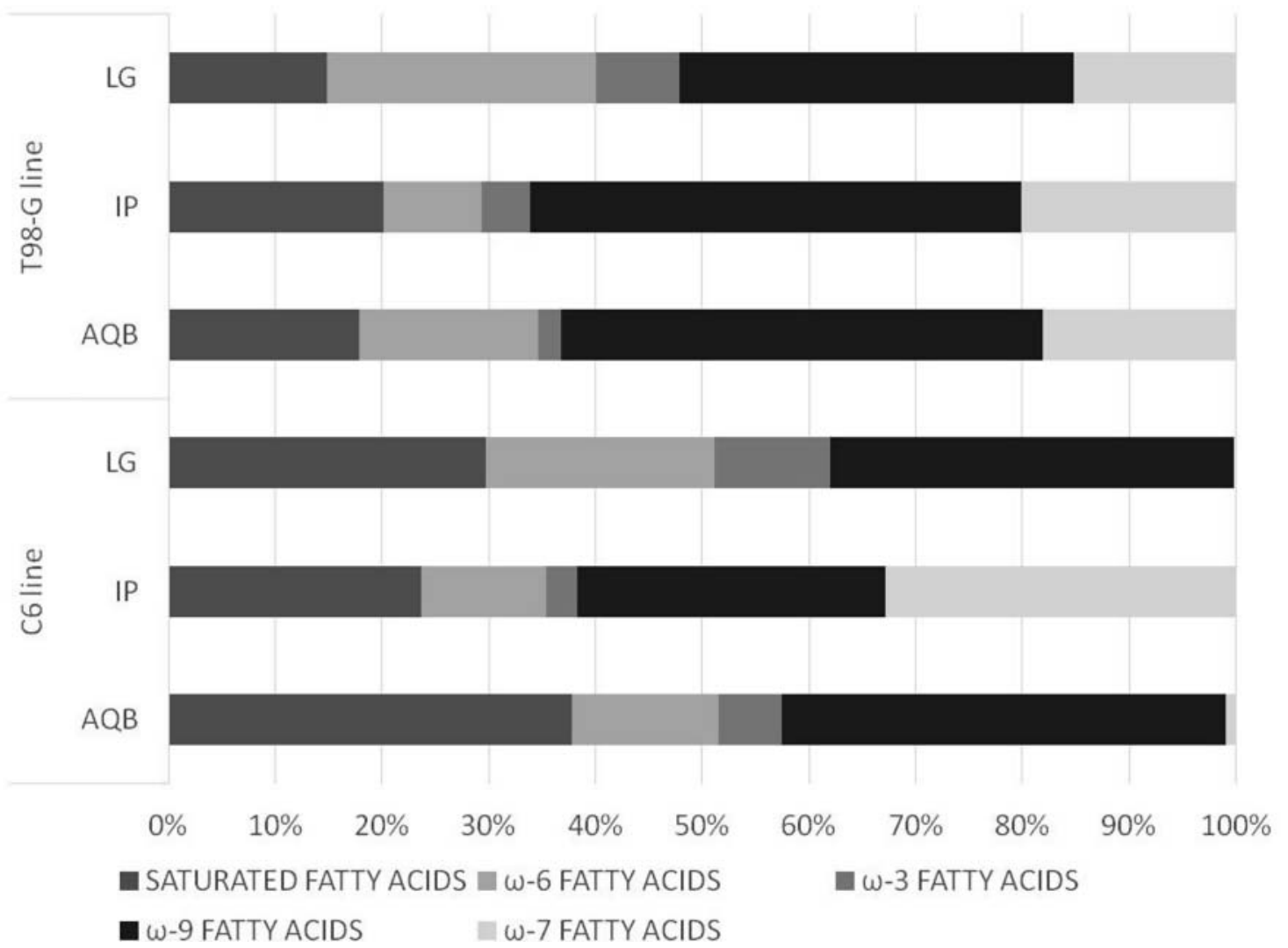

Figure 2. Fatty acid families of the C6 and T98-G cell lines treated with LG, AQB, and IP. Descriptive bars showed relative content of each one (\%).

discriminate effects of each compound. Levels of IL-6 release were inversely related to levels of $\omega-7$ fatty family, which was represented by the palmitoleic acid (Figure 3 ).

Compounds of LG were related to polyunsaturated $\omega-6$ and $\omega-3$ fatty acids, without further association with IL-6 release. Compounds of AQB, with T98-G cells being responsive, were directly related to IL- 6 release and less strongly to $\omega-9$ and saturated fatty acids (Figure 3 ).

Compounds of IP were mainly related to $\omega-7$ levels, followed by $\omega-9$ and saturated acids. Also, these compounds were inversely related to IL-6 release. The chlorogenic acid was identified as the main active compound on C6 cells, among the major phenolic acids of IP (1-caffeoylquinic, 5-caffeoylquinic, 1,3-dicaffeoylquinic) (Figure 3).

\section{Discussion}

This study analyzed numerous phenolic compounds in tea extracts of three South American plants: LG, AQB, and IP, which were assayed in cultures of two cell lines (murine C6 and human T98-G). These cells were chosen given glia plays a key role in IL-6-mediated neuroinflammation, which is involved in glioma response (23).

Concerning their chemical composition, twenty-one compounds were measured in LG, which were mainly iridoids such as dihydrotheveside and theveside. This deepens our knowledge of the genus, given that the scientific literature is often about compounds of L. camara, e.g., lantadenes (24). Moreover, the extract was a source of geniposidic derivatives, which have been described in different taxonomic families (25). Detection of verbascoside isomer, another major LG compound, was in accordance with phytochemical profile of Verbenaceae (26). Although some iridoids reduce inflammatory responses (27), LG compounds did not modify IL-6 in this work. Nonetheless, they were active and positively related to cellular content of polyunsaturated fatty acids, which was previously found [unpublished data].

Thirty-one compounds were found in $\mathrm{AQB}$, which mainly contained hydroxybenzoic acid derivatives. It also presented different flavonoids, such as isorhamnetin glycosides. These results are original because previous research generally focused on its alkaloids (28). Although the gallic acid is widely distributed in Plantae, the major compounds trihydroxy-phthalic acid hexoside and gentisoyl-dihexoside are less frequent and found in certain families (29,30). Thus, phytochemical profile of AQB was extended by this work. Other minor compounds were in accordance with phytochemical profile of Apocynaceae (30). Although some minor compounds such as isorhamnetin exhibit anti-inflammatory potential (31), 


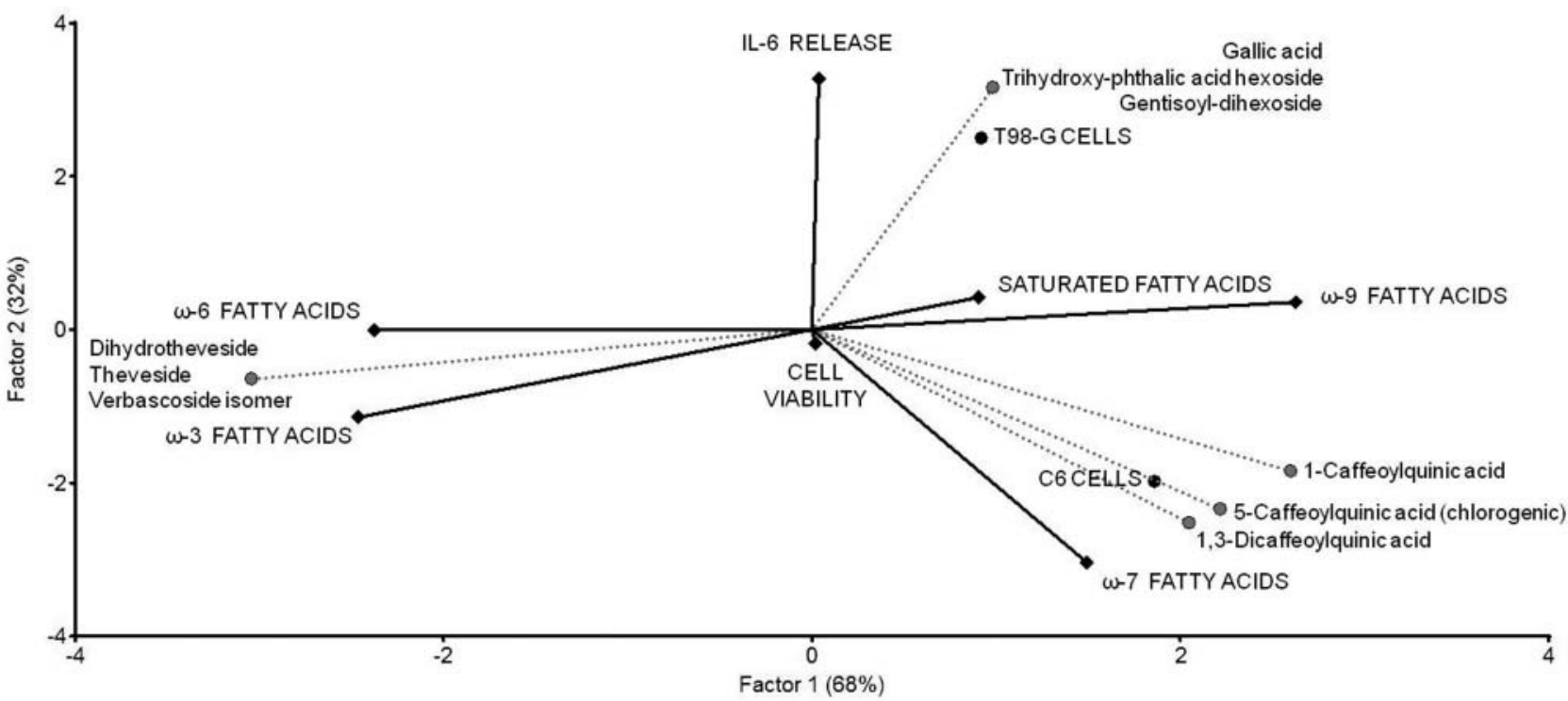

Figure 3. Response correlation according to extract compound doses (major compounds: LG: dihydrotheveside, theveside, verbascoside isomer; AQB: gallic acid, trihydroxy-phthalic acid hexoside, gentisoyl dihexoside; IP: chlorogenic acid, 1-caffeoylquinic acid, 1,3-dicaffeoylquinic acid) in the $\mathrm{C} 6$ and T98-G cell lines. Line length indicated relative variable intensity, and grades of line separation indicated variable correlation (i.e., $<90^{\circ}=$ positive, $90^{\circ}=$ null, $>90^{\circ}=$ negative).

role of major $\mathrm{AQB}$ compounds in neuroinflammation is unknown. Actually, the extract promoted IL-6 release in this work.

In the case of IP, the infusion provided more compounds in amount per $\mathrm{ml}$ than the others ( $>\mathrm{LG}>\mathrm{AQB}$ ). IP presented twenty-three identifiable compounds, including several caffeoylquinic acid derivatives. Although molecules of this plant have previously been identified $(6,32)$, the current work found a remarkable content of chlorogenic acid. The reduction of IL- 6 release found after exposure to these phenolic acids (1-, 5-, and 1,3- forms) was according to their anti-inflammatory activity $(32,33)$.

The effects of IP compounds were mostly associated with the increase of $\omega-7$ fatty family, represented by the palmitoleic acid. It therefore confirmed the anti-inflammatory effect of this fatty acid on glial cells, including reduction of IL-6 and other inflammatory mediators found in other cells $(34,35)$. In addition to this activity, IL-6 is also involved in several biological processes in the brain, as regulator of synaptic plasticity, neural networks, cognitive responses and development of neuropathies (36). This indicated a regulatory role of phenolic acids on glial lipid metabolism (i.e. chlorogenic acid might upregulate stearoyl-9-CoA desaturase, which was supported by $\omega-9$ levels). This gives a new insight into a poorly understood fatty acid and its role as a target of neuro-immunomodulating agents. Furthermore, neuroprotective signalling promotes palmitoleic acid (37), with content of this $\omega$-7 lipid being enhanced in murine brain by the IP infusion (38).
Polyphenols provided by each infusion can rise $\sim 10 \%$ to $25 \%$ of total daily polyphenols after being included into the diet, in accordance with results shown in Tables 1-3 and revised data (39), which supports their relevance. Moreover, IP concentration used in cultures provided relevant levels of chlorogenic acid, which has been detected in brain after oral consumption of the extract [unpublished data], under representative conditions of human intake (6).

\section{Conclusion}

This study provides a broad phytochemical characterization of three South American plants, which contain neuroactive molecules with importance for human nutrition and health. IP is commonly used as a tea called "mate," which provides high amounts of dietary phenolic compounds with anti-inflammatory activity on glioma cells, whereas LG and AQB did not. Also, results indicate that these compounds modify the lipid composition of glia and its immune function, with a specific role of the palmitoleic acid being found by the current work.

\section{Acknowledgments}

This study was financially supported by the Universidad Nacional de Córdoba (grant $\mathrm{N}^{\circ} 313 / 2016$, Argentina), and the Ministerio de Economía y Competitividad (project ref. AGL2014-58486-C2-1-R, co-financed with FEDER, Spain). The funders had no role in study design, data collection and analysis, decision to publish, or preparation of the manuscript. 
The authors have declared that no competing interests exist, and that financial supports for this work have not influenced its outcomes.

\section{Funding}

Ministerio de Economía y Competitividad (AGL2014-58486C2-1-R); Universidad Nacional de Córdoba (313/2016).

\section{ORCID}

Elio A. Soria (iD http://orcid.org/0000-0003-2373-2493

\section{References}

1. Tsao R: Chemistry and biochemistry of dietary polyphenols. Nutrients 2, 1231-1246, 2010.

2. Madi N, Dany M, Abdoun S, and Usta J: Moringa oleifera's nutritious aqueous leaf extract has anticancerous effects by compromising mitochondrial viability in an ROS-dependent manner. J Am Coll Nutr 35(7), 604-613, 2016.

3. Canalis AM, Cittadini MC, Albrecht C, and Soria EA: In vivo redox effects of Aspidosperma quebracho-blanco, Lantana grisebachii and Ilex paraguariensis on blood, thymus and spleen of mice. Indian J Exp Biol 9(52), 882-889, 2014.

4. Cittadini MC, Canalis AM, Albrecht C, and Soria EA: Effects of oral phytoextract intake on phenolic concentration and redox homeostasis in murine encephalic regions. Nutr Neurosci 7(18), 316-322, 2015.

5. Barboza GE, Cantero JJ, Núñez C, Pacciaroni A, Ariza Espinar L: Medicinal plants: a general review and a phytochemical and ethnopharmacological screening of the native Argentine Flora. Kurtziana 1-2(34), 7-365, 2009.

6. Bracesco N, Sanchez AG, Contreras V, Menini T, and Gugliucci A: Recent advances on Ilex paraguariensis research: minireview. J Ethnopharmacol 136(3), 378-384, 2011.

7. Jin-Woo J, Won SL, Sung CS, Gi-Young K, and Byung TC: Anthocyanins downregulate lipopolysaccharide-induced inflammatory responses in BV2 microglial cells by suppressing the NF- $\kappa \mathrm{B}$ and Akt/MAPKs signaling pathways. Int J Mol Sci 14(1), 1502-1515, 2013.

8. Paolini A, Curti V, Pasi F, Mazzini G, Nano R, et al.: Gallic acid exerts as protective or an anti-proliferative effect on glioma T98G cells via dose-dependent epigenetic regulation mediated by miRNAs. Int J Oncol 46, 1491-1497, 2015.

9. El Mohsen M, Kuhnle G, Rechner R, Schroeter H, Rose S, et al.: Uptake and metabolism of epicatechin and its access to the brain after oral ingestion. Free Rad Biol Med 12(33), 1693-1702, 2002.

10. Dion C, Haug C, Guan H, Ripoll C, Spiteller P, et al.: Evaluation of the anti-inflammatory and antioxidative potential of four fern species from China intended for use as food supplements. Nat Prod Commun 10(4), 597-603, 2015.
11. Kyritsis AP, Bondy ML, and Levin VA: Modulation of glioma risk and progression by dietary nutrients and antiinflammatory agents. Nutr Cancer 63(2), 174-184, 2011.

12. Alcalde-Eon C, García-Estévez I, Ferreras-Charro R, RivasGonzalo JC, Ferrer-Gallego R, et al.: Adding enological tannins vs. overripe grapes: effect on the phenolic composition of red wines. J Food Comp Anal 34, 99-113, 2014.

13. García-Estévez I, Escribano-Bailón MT, Rivas-Gonzalo JC and Alcalde-Eon C: Development of a fractionation method for the detection and identification of oak ellagitannins in red wines. Anal Chim Acta 660, 170-176, 2010.

14. García-Estévez I, Escribano-Bailón MT, Rivas-Gonzalo JC and Alcalde-Eon C: Validation of mass spectrometry method to quantify oak ellagitannins in wine samples. $J$ Agric Food Chem 60, 1373-1379, 2012.

15. Clifford MN, Luu W, Kirkpatrick J, and Kuhnert N: Profiling the chlorogenic acids and other caffeic acid derivatives of herbal chrysanthemum by LC-MS. J Agric Food Chem 55, 929-936, 2007.

16. Sousa EO, Miranda CM, Nubre CB, Boligon AA, and Athayde ML: Phytochemical analysis and antioxidant activities of Lantana camara and Lantana montevidensis extracts. Ind Crops Prod 70, 7-15, 2015.

17. Soria EA, Quiroga PL, Albrecht C, Ramos Elizagaray SI, Cantero JJ, et al.: Development of an antioxidant phytoextract of Lantana grisebachii with lymphoprotective activity against in vitro arsenic toxicity. Adv Pharmacol Sci 2014, 416761, 2014.

18. Folch J, Lees M, and Sloane S: A simple method from the isolation and purification of total lipides from animal tissues. J Biol Chem 226, 497-509, 1957.

19. Comba A, Maestri DM, Berra MA, Garcia CP, Das UN, et al.: Effect of $\omega-3$ and $\omega-9$ fatty acid rich oils on lipoxygenases and cyclooxygenases enzymes and on the growth of a mammary adenocarcinoma model. Lipids Health Dis 9, 112, 2010.

20. Martens H, Anderssen E, Flatberg A, Gidskehaug LH, Westad F, et al.: Regression of a data matrix on descriptors of both its rows and of its columns via latent variables: LPLSR. Comput Stat Data Anal 48, 103-123, 2005.

21. Sæbø S, Almøy T, Flatberg A, Aastveit AH, and Martens H: LPLS-regression: a method for prediction and classification under the influence of background information on predictor variables. Chemometr Intell Lab 91, 121-132, 2008.

22. Ferreres F, Grosso C, Gil-Izquierdo A, Valentao P, Azevedo $\mathrm{C}$, et al.: HPLC-DAD-ESI/MSn analysis of phenolic compounds for quality control of Grindelia robusta Nutt. and bioactivities. J Pharm Biomed Anal 94, 163-172, 2014.

23. Liu X, Yang J, and Deng W: The inflammatory cytokine IL-22 promotes murine gliomas via proliferation. Exp Ther Med 13, 1087-92, 2017.

24. Grace-Lynn C, Darah I, Chen Y, Yoga Latha L, and Jothy SL: In vitro antioxidant activity potential of lantadene A, a pentacyclic triterpenoid of Lantana plants. Molecules 17, 11185-11198, 2012.

25. Xu P, Du S, Lu Y, Bai J, Liu H, et al.: UPLC-MS/MS determination of content of three iridoids of xingnaojing oral preparation in rat brains and study on their brain pharmacokinetics. Zhongguo Zhong Yao Za Zhi 39(12), 23512355, 2014.

26. Leitão GG, Pinto SC, de Oliveira DR, Timoteo $P$, Guimarães MG, et al.: Gradient X isocratic elution CCC 
on the isolation of verbascoside and other phenylethanoids: influence of the complexity of the matrix. Planta Med 81, 1609-1613, 2015.

27. Park KS, and Kim BH: II-Moo C: Inhibitory potencies of several iridoids on cyclooxygenase-1, cyclooxygenase-2 enzymes activities, tumor necrosis factor- $\alpha$ and nitric oxide production in vitro. eCAM 7, 41-45, 2010.

28. Layne $\mathrm{T}$, Roach $\mathrm{J}$, and Tinto $\mathrm{W}$ : Review of $\beta$-carboline alkaloids from the genus Aspidosperma. Nat Prod Commun 10, 183-186, 2015.

29. Barrajón-Catalán E, Fernández-Arroyo S, Roldán C, Guillén E, Saura D, et al.: A systematic study of the polyphenolic composition of aqueous extracts deriving from several Cistus genus species: evolutionary relationship. Phytochem Anal 22, 303-312, 2011.

30. Ezuruike UF, and Prieto JM: The use of plants in the traditional management of diabetes in Nigeria: pharmacological and toxicological considerations. J Ethnopharm 55, 857-924, 2014.

31. Antunes-Ricardo M, Gutierrez-Uribe JA, Martinez-Vitela C, and Serna-Saldívar SO: Topical anti-inflammatory effects of isorhamnetin glycosides isolated from Opuntia ficus-indica. Biomed Res Int 2015, 847320, 2015.

32. Puangpraphant S, Berhow MA, Vermillion K, Potts G, Gonzalez de Mejia E: Dicaffeoylquinic acids in Yerba mate (Ilex paraguariensis St. Hilaire) inhibit NF- $\kappa \mathrm{B}$ nucleus translocation in macrophages and induce apoptosis by activating caspases- 8 and -3 in human colon cancer cells. Mol Nutr Food Res 55(10), 1509-1522, 2011.
33. Vera F, Costa G, Figueirinha A, Marques C, Pereira P, et al.: Anti-inflammatory activity of Cymbopogon citratus leaves infusion via proteasome and nuclear factor-KB pathway inhibition: contribution of chlorogenic acid. $J$ Ethnopharm 148, 126-134, 2013.

34. Chan K, Pillon N, Sivaloganathan D, Costford S, Liu Z, et al.: Palmitoleate reverses high fat-induced pro-inflammatory macrophage polarization via AMP-activated protein kinase (AMPK). J Biol Chem 290(27), 16979-16988, 2015.

35. Macrae K, Stretton C, Lipina C, Blachnio-Zabielska A, Baranowski $\mathrm{M}$, et al.: Defining the role of DAG, mitochondrial function, and lipid deposition in palmitateinduced pro-inflammatory signaling and its countermodulation by palmitoleate. J Lip Res 54(9), 23662378, 2013.

36. Levin SG, and Godukhin OV: Modulating effect of cytokines on mechanisms of synaptic plasticity in the brain. Biochemistry (Mosc) 82(3), 264-274, 2017.

37. Suzuki S, Hongli Q, Okada A, Kasama T, Ohta K, et al.: BDNF-dependent accumulation of palmitoleic acid in CNS neurons. Cell Mol Neurobiol 32, 1367-1373, 2012.

38. Cittadini MC, and Soria EA: Chemoprevention of the neurooncological development by redox-active phytoextracts of autochthonous species Spanish. In: INC. Informes finales tercera cohorte de becarios. Buenos Aires: INC, 2015, pp. 39-49.

39. Scalbert A, and Williamson G: Dietary intake and bioavailability of polyphenols. J Nutr 130(8S Suppl), 2073S-2085S, 2000. 